\title{
Conjectures on Non-Local Effects in String Black Holes
}

\author{
B.Harms and Y.Leblanc \\ Department of Physics and Astronomy, The University of Alabama \\ Box 870324, Tuscaloosa, AL 35487-0324
}

\begin{abstract}
We consider the modifications to general relativity by the non- local (classical and quantum) string effects for the case of a D-dimensional Schwarzschild black hole. The classical non-local effects do not alter the spacetime topology (the horizon remains unshifted, at least perturbatively). We suggest a simple analytic continuation of the perturbative result into the non- perturbative domain, which eliminates the black hole singularity at the origin and yields an ultraviolet-finite theory of quantum gravity. We investigate the quantum non-local effects (including massive modes) and argue that the inclusion of these back reactions resolves the problem of the thermal spectrum in the semi- classical approach of field quantization in a black hole background, through the bootstrap condition. The density of states for both the quantum and thermal interpretation of the WKB formula are finally shown to differ quantitatively when including the non-local effects.
\end{abstract}

PACS numbers: 4.60.+n, 11.17.+y, 97.60.lf

Typeset Using REVTEX 


\section{INTRODUCTION}

In previous works [1], we have shown that the semiclassical (WKB) approximation of the Euclidean path integral of a quantized theory of gravity in a black hole background should properly be interpreted as the probability of quantum tunneling of a particle across the black hole horizon barrier. This interpretation yielded directly the quantum black hole degeneracy of states and resolved all the problems associated with the traditional thermodynamical interpretation in a way consistent with the laws of thermodynamics and quantum mechanics. We were then led to the "finger-printing" of D-dimensional neutral black holes through their degeneracy of states and to their identification as quantum $\frac{(D-2)}{(D-4)}$-branes.

Although our work has shed some light on the quantum black hole problem, still questions remain unanswered. Of particular urgency is the classic problem of field quantization in the classical black hole background. It has been demonstrated over and over again that "naive quantization" of fields in such a background leads to an exact thermal distribution for the particle number density with the (Hawking) temperature related to the mass of the black hole, in agreement with the WKB approximation calculation.

It has often been argued, and extensively investigated in various models and dimensions, that quantum gravity effects (loop corrections or back reaction effects) would actually resolve the problem of the thermal spectrum. That it is indeed a problem is related to the prediction that a black hole having absorbed initially particles in pure states will emit them at later times in a thermal (mixed) state during its evaporation process, thereby violating the law of quantum mechanics asserting that pure states evolve solely into pure states in any timedependent process.

In this paper, we intend to investigate the nature of the above problem through a series of (qualitative) arguments, partially supported by explicit computations.

It is important to get a headstart on this problem and its resolution by first accepting a present-day fact about quantum gravity, namely, the only known "consistent" theories of quantized gravity are those of quantum p-brane theories. In this work, as a simple working 
model, we shall investigate critical bosonic string (1-brane) theory compactified down to D dimensions. With the acceptance of such theories as quantum gravity theories, comes the realization that general relativity (GR) now becomes doubly modified, once through higher derivative (non-local) classical contributions arising fundamentally from the intrinsic nonlocal nature of p-branes, and once from purely quantum (loop) corrections, the so-called back-reaction effects, which must include the higher massive string (or p-brane) excitations. In this work we study the latter qualitatively and the former quantitatively.

Non-local effects, such as (Riemann) curvature-square corrections to Einstein's equations, have been considered by previous authors [5]. We review such calculations in the following section by applying perturbation theory about the D-dimensional Schwarzschild black hole metric. The small perturbation parameter is taken to be the Regge slope $\alpha^{\prime}=\frac{1}{2 \pi T}$, where $T$ is the string tension. The non-local $\alpha^{\prime}$-corrections were found [6] not to modify Hawking's black hole thermodynamics in any significant way. The horizon (topological) structure of the black hole spacetime remains undisturbed by these non-local effects, at least perturbatively.

Pending plausible analytical continuations to strong $\alpha^{\prime}\left(\alpha^{\prime} \rightarrow \infty\right)$, we show that the black hole singularity at the origin can be eliminated. So, non-local effects remove the ultraviolet divergences of quantum gravity in all Feynman loop diagrams involving graviton propagators, a purely stringy effect directly related to the removal of the black hole singularity $(r \rightarrow 0)$. With such corrections, gravity is shown to vanish at the black hole center (asymptotic freedom). Although the Hawking temperature is in general modified by the $\alpha^{\prime}$-corrections, Green's functions of quantized fields in the modified black hole background remain periodic with respect to the (modified) inverse Hawking temperature (Kubo-MartinSchwinger property) and so again lead to an exact thermal spectrum for the particle number density, even though the black hole singularity is removed.

Perturbatively, the classical stringy corrections to Einstein's equations do not disturb the global topology of the spacetime. This illustrates the point that the seemingly thermal property of black holes is not intrinsic to the black hole as a curvature- singular object. In fact, any regular spacetime, whether curved or not and which possesses an horizon, leads to 
an exact thermal spectrum (e.g. static de Sitter space, Rindler space, etc.).

Clearly the resolution of the problem of the exact thermal spectrum must lie within the quantum effects. Fundamentally, the origin of the Hawking's radiation (thermal spectrum) phenomenon lies in the fact that the horizon acts as an impenetrable barrier between classically causally disconnected sectors of spacetime and that, although a quantized field may exist everywhere in the spacetime, information on the field from sectors not attainable by an observer is seemingly lost.

Finding the true vacuum is a highly non-trivial exercise and is truly a self-consistent (nonperturbative) problem in which the classical background determines the quantization process which itself determines this same background.

In the last section, we show how the bootstrap condition inherent in the quantum (tunneling probability) interpretation of the WKB formula leads to a possible (non-perturbative) solution of the thermal spectrum problem.

\section{STRING CORRECTIONS TO BLACK HOLE BACKGROUNDS}

Corrections to the metric tensor due to 'stringy' (non- local) classical effects were carried out to $O\left(\alpha^{\prime}\right)$ in Ref. [6]. For a bosonic string the usual form for the action

$$
S_{0}=\frac{1}{16 \pi} \int d^{D} x \sqrt{-g} e^{-2 \phi}\left(R+4(\nabla \phi)^{2}+\frac{\alpha^{\prime}}{4} R_{k l m n} R^{k l m n}\right)
$$

where $D$ is the dimension of the black hole, gives the equations of motion for the graviton and the dilaton

$$
\begin{aligned}
R_{i j}+2 \nabla_{i} \nabla_{j} \phi+\frac{\alpha^{\prime}}{2} R_{i k l m} R_{j}^{k l m} & =0, \\
\square \phi-(\nabla \phi)^{2}+\frac{1}{4} R+\frac{\alpha^{\prime}}{16} R_{k l m n} R^{k l m n} & =0 .
\end{aligned}
$$

The action given in Eq.(2.1) leads to a difference between the inertial and gravitational masses of the black hole due to the coupling of the dilaton to the trace of the graviton. This problem can be overcome by making the conformal transformation [6], 


$$
g_{i j} \rightarrow \exp \left(\frac{4}{D-2} \phi\right) g_{i j}
$$

This transformation, together with some field redefinitions, changes the action to,

$$
S^{\prime}=\frac{1}{16 \pi} \int d^{D} x \sqrt{-g}\left(R-\frac{4}{D-2}(\nabla \phi)^{2}+\frac{\alpha^{\prime}}{4} e^{-4 \phi /(D-2)} R_{k l m n} R^{k l m n}\right)
$$

The resulting equations of motion

$$
\begin{aligned}
R_{i j}+\frac{\alpha^{\prime}}{2} e^{-4 \phi /(D-2)}\left(R_{i k l m} R_{j}^{k l m}-\frac{1}{2(D-2)} g_{i j} R_{k l m n} R^{k l m n}\right) & =0, \\
\square \phi-\frac{\alpha^{\prime}}{8} e^{-4 \phi /(D-2)} R_{k l m n} R^{k l m n} & =0,
\end{aligned}
$$

can be solved perturbatively by expanding about the $\alpha^{\prime}=0$ Schwarzschild metric for a static black hole and about the constant part of the dilaton field. To first order in $\alpha^{\prime}$ the elements of the metric tensor and the dilaton field are given by

$$
\begin{array}{r}
g_{00}=g_{00}^{(0)}\left(1+\alpha^{\prime} f(r)\right), \\
g_{11}=g_{11}^{(0)}\left(1+\alpha^{\prime} g(r)\right), \\
g_{i i} d x_{i}^{2}=r^{2} d \Omega_{D-2}^{2} ; i \geq 2, \\
\phi=\phi_{0}+\frac{\alpha^{\prime}}{2} \varphi(r),
\end{array}
$$

where

$$
g_{00}^{(0)}=\left(-g_{11}^{(0)}\right)^{-1}=-\left(1-\frac{\kappa}{r^{D-3}}\right)
$$

$d \Omega_{D-2}^{2}$ is a line element on the unit $D-2$-sphere, and $\phi_{0}$ is an arbitrary constant. $\kappa$ is related to the unperturbed mass of the black hole by,

$$
m=\frac{(D-2) A_{D-2}}{16 \pi} \kappa
$$

where $A_{D}$ is the area of a unit D-sphere. Using these expressions in Eq.(2.6) it is found that 6),

$$
f=-g,
$$




$$
g=\frac{(D-3)(D-4)}{4} \frac{r_{+}^{D-5}}{r^{D-1}} \frac{r^{D-1}-r_{+}^{D-1}}{r^{D-3}-r_{+}^{D-3}},
$$

and $\varphi$ satisfies the differential equation

$$
\frac{d \varphi}{d r}=\frac{(D-2)^{2}(D-3)}{4} \frac{r_{+}^{D-5}}{r^{D}} \frac{r^{D-1}-r_{+}^{D-1}}{r^{D-3}-r_{+}^{D-3}},
$$

$r_{+}$being the value of $r$ at the horizon, $r_{+}=\kappa^{\frac{1}{D-3}}$. These expressions show that to first order in $\alpha^{\prime}$ there are no corrections to the metric tensor elements in four dimensions. However in higher dimensions and for higher orders in the $\alpha^{\prime}$ expansion in four dimensions the metric tensor is modified and hence the action and the thermodynamical quantities calculated from it are also modified.

\section{STRING CORRECTIONS TO THE THERMODYNAMICS OF BLACK HOLES}

Non-local contributions to the metric tensor will affect the thermally interepreted character of the geometry. According to Gibbons and Hawking [8], the thermodynamical properties of a black hole with inverse temperature $\beta_{H}$ can be obtained by analytically continuing the metric to Euclidean spacetime, and summing over geometries which are asymptotically flat, have topology $R^{2} \times S^{2}$ and are periodic in imaginary time $\tau$ with period $\beta_{H}$. In bosonic string theory the spacetime dimension is 26 , so that we consider a manifold which is a direct product of a $D$-dimensional black hole and a $26-D$ - dimensional compact internal space. The thermodynamical quantities of interest are obtained from the partition function, which in the usual semiclassical (WKB) approximation is given by,

$$
Z\left(\beta_{H}\right) \sim \exp \left(-S_{E} / \hbar\right)
$$

where $S_{E}$ is the Euclidean action evaluated at the black hole solution. The total Euclidean action is the sum of the analytically continued action of Eq.(2.5) and a boundary term $S_{b d}$ (see Ref.[6]).

As usual $\beta_{H}$ is the inverse Gibbons-Hawking temperature given by, 


$$
\beta_{H}=\frac{2 \pi}{\left[\left(\partial_{r} e^{\Phi(r)}\right) e^{-\Lambda(r)}\right]_{r=r_{+}}} .
$$

where the functions $\Lambda(r)$ and $\Phi(r)$ are given by

$$
\begin{aligned}
& \Lambda(r)=\frac{1}{2} \ln \left(g_{11}\right), \\
& \Phi(r)=\frac{1}{2} \ln \left(g_{00}\right) .
\end{aligned}
$$

$S_{b d}$ is to be evaluated at spatial infinity but actually diverges as $r \rightarrow \infty$. The corresponding action of a flat spacetime at the boundary must be subtracted [8] to eliminate this divergence. The total Euclidean action is

$$
\begin{aligned}
S_{E} & =S+S_{b d}-S_{b d}^{\text {flat }}, \\
& =\frac{A_{D-2}}{16 \pi} \beta_{H} r_{+}\left(1-(D-2)(D-3) \frac{\alpha^{\prime}}{2 r_{+}^{2}}\right) .
\end{aligned}
$$

To order $\alpha^{\prime}$ the inverse temperature $\beta_{H}$ is given by

$$
\beta_{H}=\frac{4 \pi r_{+}}{D-3}\left(1+\frac{(D-2)(D-4)}{2} \frac{\alpha^{\prime}}{2 r_{+}^{2}}\right)
$$

The entropy to the same order in $\alpha^{\prime}$ is

$$
\begin{aligned}
S & =\beta_{H}\langle E\rangle-S_{E} \\
& =\frac{A_{D-2}}{4} r_{+}^{D-2}\left(1+\frac{(D-2)^{2}}{2} \frac{\alpha^{\prime}}{2 r_{+}^{2}}\right) .
\end{aligned}
$$

where $\langle E\rangle=M$, the $\alpha^{\prime}$-corrected mass of the black hole [6],

$$
M=m\left[1+\frac{\alpha^{\prime}(D-3)(D-4)}{4 r_{+}^{2}}\right]
$$

Eq.(3.6) shows that stringy effects modify the area law. However the first law of black hole thermodynamics

$$
\beta_{H}=\frac{d S}{d M}=8 \pi M
$$

is still satisfied. 


\section{ULTRAVIOLET FINITE QUANTUM GRAVITY}

The content of this section is primarily based upon the Limiting Curvature Hypothesis (LCH) [12] which states that no classical curvature singularity should occur in a suitable geometrical gravitational theory. This is required in order for the spacetime to be geodesically complete. This principle effectively provides for the realization of Penrose's Cosmic Censorship Hypothesis.

Another important input into the working assumptions of this section is the belief that the non-local p-brane theories (including of course string theories) are ultraviolet (UV) finite theories.

As is clear from the viewpoint of the solution Eqs.(2.7),(2.10) for the black hole metric incorporating the non-local effects to first order in $\alpha^{\prime}$, the singular behavior at the origin of the metric worsens as we go to higher orders in $\alpha^{\prime}$-perturbation expansion. That it is so originates from the fact that the higher order terms in the corrected Einstein's equations have an increasingly higher number of derivatives. The perturbation series in $\alpha^{\prime}$ is therefore a series of individually divergent terms as $r \rightarrow 0$. However, in view of the arguments of the preceding paragraphs, the series itself should be regular as $r \rightarrow 0$. To uncover the full solution would probably require a great deal more calculation involving the higher order terms.

For our purposes however, a simple analytic continuation of the perturbative results to strong $\alpha^{\prime}$ (although it is by no means unique) will suffice. Let us consider the following analytical continuation to strong $\alpha^{\prime}$ (or small $r$ ),

$$
g_{o o}=-1+\frac{r_{+}^{D-3}}{r^{D-3}} \exp \left[\alpha^{\prime} C\left(\frac{1}{r_{+}^{D-1}}-\frac{1}{r^{D-1}}\right)\right] .
$$

where we defined $C \equiv \frac{(D-3)(D-4) r_{+}^{D-3}}{4}$.

Notice that the horizon of this spacetime is again left undisturbed by the $\alpha^{\prime}$-effects.

It is now apparent that the singularity at the origin has been removed. Indeed spacetime is flat at the origin. Now since $g_{o o}=-1-2 U(r)$, in which $U(r)$ is the gravitational potential, 
Eq.(4.1) gives the following stringy modification to Newton's gravity,

$$
U(r) \propto-\frac{M}{r^{D-3}} \exp \left[\frac{-\alpha^{\prime} C}{r^{D-1}}\right] .
$$

It may be useful to comment here that the non-local $\alpha^{\prime}$-corrections to Newton's law will produce modifications (although very small) to phenomena such as the gravitational redshift, the bending of light by the sun and the perihelion of Mercury. If refined experiments were carried out (which may be decades or centuries away), these would allow for the first direct measurements of the string tension.

Let us now express the Fourier transform of the gravitational potential $U(r)$ as follows,

$$
U(k) \equiv-\frac{M}{A_{D-2}} \int_{-\infty}^{\infty} d^{D-1} \vec{x}\left\{|\vec{x}|^{3-D} \exp \left[\frac{-\alpha^{\prime} C}{|\vec{x}|^{D-1}}\right]\right\} e^{i \vec{k} \cdot \vec{x}} ;(k=|\vec{k}|)
$$

We find,

$$
U(k)=-M \lim _{\epsilon \rightarrow 0} \int_{0}^{\infty} d r r^{1-\epsilon} \frac{\sin k r}{k r} \exp \left[\frac{-\alpha^{\prime} C}{r^{D-1}}\right]
$$

in which $\epsilon$ is an infrared (IR) $(r \rightarrow \infty)$ regulator.

Introducing the dimensionless parameter $\sigma \equiv k r$, we finally arrive at the following explicit integral representation for the graviton "propagator" $U(k)$,

$$
U(k)=-\frac{M}{k^{2}} \lim _{\epsilon \rightarrow 0} \int_{0}^{\infty} d \sigma \frac{\sin \sigma}{\sigma^{\epsilon}} \exp \left[\frac{-\alpha^{\prime} C k^{D-1}}{\sigma^{D-1}}\right] .
$$

Two important remarks must be made. First, as is clear from the above formula, the IR behavior of the graviton propagation function remains unaltered by the (re-summed) nonlocal $\alpha^{\prime}$-effects. The graviton remains a massless particle and no ghost particles occur in this expression. The second remark of course concerns the UV behavior of the propagator, which clearly vanishes exponentially as $k \rightarrow \infty$, at least for $D>4$. In 4 dimensions, the corrections to the propagator are of order $O\left(\alpha^{\prime 2}\right)$ [7]. It is now obvious that any loop Feynman diagrams involving internal graviton propagators lead to UV-finite results. Loop diagrams should be evaluated by first integrating over the loop momenta, then integrating over the integral representation parameters $\sigma_{i}$ 's and finally taking the $\epsilon_{i}$ 's IR regulators to zero, in that order. 
Of course these apply to the Feynman rules of a field theory of gravity, namely quantum GR, which itself is the $\alpha^{\prime} \rightarrow 0$ limit of string theory. The above considerations show that not all $\alpha^{\prime}$-effects should be taken to zero in order to recover the field theory limit, if finite predictions are to be made from such a theory.

\section{STRINGY BACK REACTION EFFECTS}

Up to this point, we addressed the problem of the effects of the classical non-local ( $\alpha^{\prime}-$ corrections) modifications of GR from the string progenitor, in connection with black hole spacetimes.

The results are that the non-local effects do not disturb the topological character of the spacetime (the horizon remains unshifted, at least perturbatively).

In addition, pending the plausible analytical continuations of the results of Section II to the strong $\alpha^{\prime}$ domain, as discussed in Section IV, we found that the quantum field theory limit (quantum GR) of string theory is UV-finite, provided some $\alpha^{\prime}$-dependency remains in the field theory limit.

On the other hand, because the non-local classical $\alpha^{\prime}$-corrections do not influence the topology of the black hole (although they do generally reduce its Hawking temperature), field quantization in the black hole background will continue to yield a thermal spectrum (with shifted Hawking temperature) for the particle number density, a somewhat disappointing result if one is to believe in the pure quantum nature of black holes.

As mentioned in the Introduction, the resolution of the thermal spectrum must originate from the quantum or back reaction effects. Attempts to resolve this problem have been made by various authors and in various numbers of dimensions.

An intuitive, if not totally naive, way to see that this may indeed occur, is the following consideration making use of the statistical bootstrap model of quantum Schwarzschild black holes [1, 2]. The situation is depicted in Fig. 1, where a single massive quantum black hole is modeled as a gas consisting itself of a single quantum massive black hole excitation sur- 
rounded by countless extreme (massless) others. Of course the equilibrium state of this gas is not thermal, as the energy distribution is highly inhomogeneous [1,2]. If for some reason the massive excitation is cut-off from the rest, the original quantum black hole will resemble a gas of massless excitations in thermal equilibrium. It is the effect of the single massive excitation in the gas which drastically changes the nature of the equilibrium configuration and allows for the bootstrap property. This massive excitation of course is a pure quantum gravity (back reaction) effect of the p-brane type. Should such effects be included in the usual semiclassical treatments, the above statistical model shows that the thermal spectrum problem would be resolved satisfactorily by yielding a picture of the original black hole as a pure state. It must be pointed out that the above possible resolution of the thermal spectrum problem is fundamentally non- perturbative as one cannot separate linearly classical and quantum effects in the WKB semiclassical approximation. This seems to imply that the usual field quantization in the black hole background may become fully consistent only after resummation of a certain class of Feynman diagrams. This is tantamount to trying to reach the superconducting phase by perturbing about the normal phase. Obviously, this type of calculation is going nowhere.

As a final comment, it may very well be sensible to expect quantum gravity effects to induce an effective cosmological constant in the original metric as loop corrections induce a non-zero vacuum energy. Consequently, it is possible that there is no such a thing as a quantum Schwarzschild black hole. The metric instead would be of the Schwarzschild-de Sitter type, which is known to contain at most two real horizons. This quantum-induced change of the topology of the black hole spacetime may have drastic effects on the thermal distribution of the particle number density already at the level of loop perturbation theory. Explicit computations are currently being carried out. 


\section{CONCLUSION}

In this work, we have looked at the modifications to the D-dimensional Schwarzschild black hole solution of GR due to stringy $\alpha^{\prime}$-effects, either classically or quantum mechanically. We have presented conjectures on how non-perturbative $\alpha^{\prime}$-effects might affect classically the black hole singularity at the origin and thus lead to its removal, as well as how nonperturbative (WKB) quantum effects might resolve the problem of the thermal spectrum. In the former case, the argumentation was quantitative in nature while the latter case was treated qualitatively.

As a final comment, let us point out that, taking into account the $\alpha^{\prime}$-corrections, one finds quantitatively different predictions for the number density of D-dimensional black holes for both the quantum and thermal interpretations. This can be seen easily seen if one make use of the following relationship between the Euclidean action and the entropy,

$$
S=(D-3) S_{E}+O\left(\alpha^{\prime}\right)
$$

Recall that $\rho=e^{S_{E}}$ while $\Omega=e^{S}$.

Our overall emerging picture of a quantum black hole is thus that of an elementary excitation mode of some quantum p-brane living in its geodesically complete regular spacetime condensate.

\section{ACKNOWLEDGMENTS}

We are grateful to J.D.Bekenstein as well as the referees of a previous version of the present manuscript for pointing out erroneous results in our perturbative treatment as well as making us aware of the work of Ref.[6].

This research was supported in part by the U.S. Department of Energy under Grant No. DE-FG05-84ER40141. 


\section{REFERENCES}

[1] B. Harms and Y. Leblanc, Phys. Rev. D46, 2334 (1992).

[2] B. Harms and Y. Leblanc, Phys. Rev. D47 2438 (1993).

[3] B. Harms and Y. Leblanc, to appear in the Proceedings of the Texas/Pascos Conference held at the University of California, Berkeley, CA (December 1992).

[4] B. Harms and Y. Leblanc, to appear in the Proceedings of SUSY-93 held at Northeastern University, Boston, MA (March 1993).

[5] M.B. Green, J.H. Schwarz and E. Witten, Superstring Theory, Vol. I, Cambridge University Press, Cambridge, 1987.

[6] C.G. Callan, R.C. Myers and M.J. Perry, Nucl. Phys. B311, 673(1988).

[7] R. Myers, Nucl. Phys. B289, 701(1987).

[8] G.W. Gibbons and S.W. Hawking, Phys. Rev. D15, 2752(1977).

[9] J.D. Bekenstein, Phys. Rev. D7, 2333(1973); Phys. Rev. D9, 3292(1974).

[10] S.W. Hawking, Phys. Rev. D13, 191(1976).

[11] S.W. Hawking, Comm. Math Phys. 43,199(1975).

[12] R. Brandenberger, V. Mukhanov and A. Sornborger, BROWN-HET-891 (1993). Unpublished. And references therein. 


\section{FIGURES}

FIG. 1. Statistical Bootstrap : $\Omega(M) \sim \rho(M) ;(M \rightarrow \infty)$. 
This figure "fig1-1.png" is available in "png" format from: http://arxiv.org/ps/hep-th/9307042v1 